\title{
A New Approach on Indoor Positioning System
}

\author{
Lina Chen ${ }^{1}$ Chunyu Miao' ${ }^{2}$ Jianmin Zhao ${ }^{1}$ \\ ${ }^{1}$ College of Mathematics, Physics and Information Engineering, Zhejiang Nor- \\ mal University, Jinhua, China \\ ${ }^{2}$ College of Xingzhi, Zhejiang Normal University, Jinhua, China
}

\begin{abstract}
Carrier phase observation equation of the inverse transmitting positioning system (ITPS) was derived. The geometric dilution of precision (GDOP) about the ITPS was also investigated. The positioning accuracy of ITPS was analyzed in detail. Indoors, on condition that there neglect multi-path and fading, by analyzing the experiments of locating a target's position in virtual three-dimensional areas, the positioning performance of IGPS was illustrated. The results show that the system in this paper can improve the positioning accuracy.
\end{abstract}

Keywords: Principle of ITPS; GDOP; Positioning Accuracy

\section{Introduction}

Recently, LBS (Location Based Services) become a large market as increased needs about location information. Location information of human and object is essential requirement for Awareness Enhancement that brings improvement of safety and efficiency in social and economic activation. Therefore location information and positioning technologies have become more and more important for implementation of location-dependent systems and services. There are varieties of technologies that can obtain location information, such as the GPS, the Pseudolite, GLONASS, Galileo, A-GPS,
Bei-Dou [1] [2] etc. It is well known that the GPS is a typical system in practical use. Although the GPS is nearly perfect for positioning in a large area, it does not work well in occasions of some bad received signal such as in environment that are inaccessible of using GPS. In order to solve this problem, the necessity of implementation of a short-haul targetlocation system which is called ITPS (inverse transmission positioning system) is increasing. There is the Inverse TPS scheme (ITPS) that differs from those positioning technologies. This may become basic technology applicable to various positioning fields, such as ubiquitous computing, positioning of a wireless LAN terminal, and this system's principle is becoming to be recognized widely. An example of the ITPS applications is positioning in a local place or positioning of a mobile terminal in the place that is unavailable for the GPS. However, some experiments with ultrasonic waves were not carried out thoroughly. Therefore, knowledge of system implementation and accuracy are still unknown.

This paper is organized as follows: Section 2 provided a scheme of the ITPS and the method of position determination. Section 3 mentioned the error factor in four stations that have influences on the positioning accuracy, and presented the fundamental experiment and discussion of the results. Conclusions and some future research works are pointed in Section 4. 


\section{The inverse transmission position- ing system}

\subsection{The IPTS scheme}

The GPS receives different pseudo noise signals from four satellites. The position of a receiver can be obtained by solving relational equations. But as shown in Fig.1, the Inverse transmission positioning system has the reverse relation between transmission and reception of GPS. The ITPS consists of one mobile transmitting station, four receiver stations, a Cable Network and a position estimation system with a phase estimation subsystem and a position calculation subsystem. The receiver station receives signals from the mobile transmitting station and transmits to the position estimation system through the cable network. The phase estimation subsystem obtains phase difference between the $i$-th receivers $(\mathrm{i}=\mathrm{j}, \mathrm{p}, \mathrm{m}, \mathrm{n})$, and position calculation subsystem calculates the position of the mobile station.

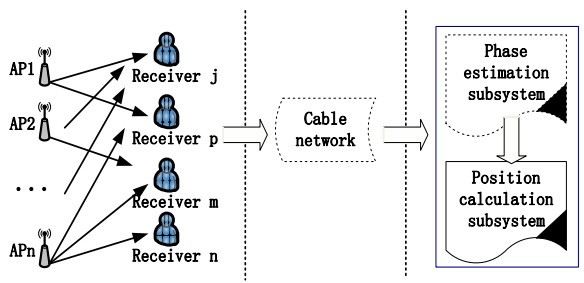

Fig. 1: The inverse transmitting positioning system.

\subsection{Principle of the ITPS}

This paper we used the method of carrier phase observation method, according to the carrier phase observation equation of GPS like Eq.1, we derived the observation equation of ITPS.

$$
\begin{aligned}
\phi & =f \cdot \frac{\sqrt{\left(x_{s}-x_{r}\right)^{2}+\left(y_{s}-y_{r}\right)^{2}+\left(z_{s}-z_{r}\right)^{2}}}{c} \\
& +\phi_{\text {trop }}+\phi_{\text {ion }}+\phi_{\text {multi }}-f\left(\tau_{r}-\tau_{s}\right)-N\left(t_{0}\right)
\end{aligned}
$$

Where $\left(x_{r}, y_{r}, z_{r}\right)$ is receiver coordinate and are unknown quantities, $\left(\mathrm{x}_{\mathrm{s}}, \mathrm{y}_{\mathrm{s}}, \mathrm{z}_{\mathrm{s}}\right)$ is satellite coordinate and are known quantities, $\phi_{\text {trop }}$ is troposphere phase error, $\phi_{\text {ion }}$ is ionosphere phase error and $\phi_{\text {multi is multi- }}$ path phase error, $f$ is frequency of transmission single, $\mathrm{c}$ is speed of light, $\mathrm{N}\left(\mathrm{t}_{0}\right)$ is ambiguity of whole cycles, $\tau_{\mathrm{r}}$ is the time difference between the accurate time $(\mathrm{Tr})$ and clock time(tr) of a receiver when the receiver received a signal from satellite: $\tau_{r}=T_{r}-t_{r}, \tau_{\mathrm{s}}$ is the time difference between the accurate time(Ts) and clock time(ts) of a satellite when the satellite transported single: $\tau_{s}=T_{s}-t_{s}$. Observed beat frequency carrier wave phase like Eq.2.

$\Phi\left(t_{r}\right)=\Phi_{r}\left(t_{r}\right)-\Phi_{s}\left(t_{s}\right)-N\left(t_{0}\right)$

It is assumed that there are not frequency drift of transmitter and receiver, then $\Phi_{r}\left(t_{s}\right)=\Phi_{s}\left(t_{s}\right)$. To Taylor expansion for $\Phi_{r}\left(t_{r}\right)=\Phi_{r}\left(T_{r}-\tau_{r}\right)$ we worked out:

$\Phi\left(t_{r}\right)=f \cdot\left(T_{r}-T_{s}\right)-f \cdot\left(\tau_{r}-\tau_{s}\right)-N\left(t_{0}\right)$

Here $\Delta T$ is accurate transmission time that single transmit from satellite to receiver.

$\Delta \mathrm{T}=\mathrm{T}_{\mathrm{r}}-\mathrm{T}_{\mathrm{s}}=\frac{\left|\overrightarrow{\mathrm{X}}_{\mathrm{r}}\left(\mathrm{T}_{\mathrm{r}}\right)-\overrightarrow{\mathrm{X}}_{\mathrm{s}}\left(\mathrm{T}_{\mathrm{s}}\right)\right|}{\mathrm{c}}+\mathrm{T}_{\text {trop }}+\mathrm{T}_{\text {ion }}+\mathrm{T}_{\text {multi }}$

$\left|\vec{X}_{r}\left(T_{r}\right)-\vec{X}_{s}\left(T_{s}\right)\right|=\rho\left(T_{s}\right)=\rho\left(T_{r}+T_{s}-T_{r}\right)=\rho\left(T_{r}-\Delta T\right)$

To Taylor expansion for Eq.5 then taken it to Eq.4, we worked out:

$\Delta T\left(T_{r}\right)=\frac{\rho\left(T_{r}\right)-\dot{\rho}\left(T_{r}\right) \cdot \Delta T}{c}+T_{\text {trop }}+T_{\text {ion }}+T_{\text {multi }}$

In order to different receivers have the same accurate time, it is assume that $\operatorname{Tr}$ is a scheduled time. So the measurement equation of ITPS is like Eq.7:

$$
\begin{aligned}
\Phi\left(t_{r}\right) & =f \cdot \Delta T\left(T_{r}\right)-f\left(\tau_{r}-\tau_{s}\right) \\
& =f \cdot \frac{\rho\left(t_{r}\right)}{c}\left(1-\frac{\dot{\rho}\left(t_{r}\right)}{c}\right)+\Phi_{\text {trop }}+\Phi_{\text {ion }}+ \\
& \Phi_{\text {multi }}-f \cdot\left(1-\frac{\dot{\rho}\left(t_{r}\right)}{c}\right) \tau_{r}+f \cdot \tau_{s}-N\left(t_{0}\right)
\end{aligned}
$$




\section{GDOP and positioning accuracy analysis}

There are several error factors which degrade the ITPS positioning accuracy. The errors can be classified two kinds. One is the multiplicative error factor, the other are additive error factors. GDOP describes the effect of geometry on the relationship between measurement error and position determination error. So GDOP is used to describe the multiplicative error factor effect to ITPS positioning performance. Most of the additive error factors cause bias error that is positioning errors with long term variations. Also some of the factors cause random error that is determined positions which appear to be scattered at random. The final positioning accuracy vale of ITPS can be expressed as the multiply by the multiplicative positioning error and additive positioning errors [3].

\subsection{The formula of GDOP}

GDOP is the main multiplicative error factor which affects the ITPS positioning accuracy. Eq. 8 is carrier phase observation equation of the ITPS.

$\tilde{\varphi}_{i} \lambda=\sqrt{\left(x_{i}-x\right)^{2}+\left(y_{i}-y\right)^{2}+\left(z_{i}-z\right)^{2}}$

$-c V_{t R}+c_{V t_{i}^{s}}-N_{i} \lambda-\left(V_{\text {ion }}\right)_{i}-\left(V_{\text {tuop }}\right)_{i}$

Where $\mathrm{cV}_{\mathrm{tR}}$ is receiver clock error, $\mathrm{c}_{\mathrm{Vt}_{\mathrm{i}}^{\mathrm{s}}}$ is satellite clock error residuals, $\mathrm{N}_{\mathrm{i}} \lambda$ is the ambiguity of whole cycles, $\left(\mathrm{V}_{\mathrm{ion}}\right)_{\mathrm{i}}$ is ionosphere delay estimation, $\left(\mathrm{V}_{\text {tuop }}\right)_{\mathrm{i}}$ is troposphere delay estimation. At time $\mathrm{t}_{1}$, receiver $\mathrm{i}$ and receiver $\mathrm{j}$ had carrier phase measurement for transmitter $\mathrm{P}$ together. $\lambda=\frac{\mathrm{c}}{\mathrm{f}}$.

$$
\left\{\begin{array}{l}
\tilde{\varphi_{i}^{p}}(t)=\frac{f}{c} p_{i}^{p}\left(t_{1}\right)-f V_{t_{i}}\left(t_{1}\right)+ \\
f V_{t p}\left(t_{1}\right)-N_{i}^{p}-\frac{f}{c}\left(V_{i o n}^{t_{1}}\right)_{i}^{p}- \\
\quad \frac{f}{c}\left(V_{\text {tuop }}^{t_{1}}\right)_{i}^{p} \\
\tilde{\varphi_{j}^{p}}(t)=\frac{f}{c} p_{j}^{p}\left(t_{1}\right)-f V_{t j}\left(t_{1}\right)+ \\
f V_{t p}\left(t_{1}\right)-N_{j}^{p}-\frac{f}{c}\left(V_{i o n}^{t_{1}}\right)_{j}^{p}- \\
\frac{f}{c}\left(V_{\text {tuop }}^{t_{1}}\right)_{j}^{p}
\end{array}\right.
$$

Where:

$\mathrm{p}_{\mathrm{i}}^{\mathrm{p}}\left(\mathrm{t}_{1}\right)=\sqrt{\left(\mathrm{x}^{\mathrm{p}}-\mathrm{x}_{\mathrm{i}}\right)^{2}+\left(\mathrm{y}^{\mathrm{p}}-\mathrm{y}_{\mathrm{i}}\right)^{2}+\left(\mathrm{z}^{\mathrm{p}}-\mathrm{z}_{\mathrm{i}}\right)^{2}}$

The time of signal send:

$$
\mathrm{t}_{1}^{\prime}=\mathrm{t}_{1}+\mathrm{V}_{\mathrm{t}_{\mathrm{i}}}\left(\mathrm{t}_{1}\right)+\frac{\mathrm{p}_{\mathrm{i}}^{\mathrm{p}}\left(\mathrm{t}_{1}\right)}{\mathrm{c}}
$$

Eq.9 subtracts Eq. 10:

$$
\begin{aligned}
& \Delta \varphi_{i j}^{p}\left(t_{1}\right)=\tilde{\varphi}_{j}^{p}\left(t_{1}\right)-\tilde{\varphi_{i}^{p}}\left(t_{1}\right)= \\
& \frac{f}{c}\left[p_{j}^{p}\left(t_{1}\right)-p_{i}^{p}\left(t_{1}\right)\right]-f\left[V_{t_{j}}\left(t_{1}\right)\right. \\
& \left.-V_{t_{i}}\left(t_{1}\right)\right]-\left(N_{j}^{p}-N_{i}^{p}\right)- \\
& \frac{f}{c}\left[\left(V_{\text {ion }}^{t_{1}}\right)_{j}^{p}-\left(V_{\text {ion }}^{t_{1}}\right)_{i}^{p}\right]- \\
& \left.\frac{f}{c}\left[V_{\text {tuop }}^{t_{1}}\right)_{j}^{p}-\left(V_{\text {tuop }}^{t_{1}}\right)_{i}^{p}\right]
\end{aligned}
$$

That is:

$$
\begin{aligned}
& \Delta \varphi_{i j}^{p}\left(t_{1}\right)=\tilde{\varphi_{j}^{p}}\left(t_{1}\right)-\tilde{\varphi_{i}^{p}}\left(t_{1}\right)= \\
& \frac{f}{c} \Delta p_{i j}^{p}\left(t_{1}\right)-f V_{t_{i j}}\left(t_{1}\right)-\Delta N_{i j}^{p}- \\
& \frac{f}{c} \Delta\left(V_{i o n}^{t_{1}}\right)_{i j}^{p}-\frac{f}{c}\left(V_{\text {tuop }}^{t_{1}}\right)_{i j}^{p}
\end{aligned}
$$

At the same ways for transmitter q,

$$
\begin{aligned}
& \Delta \varphi_{i j}^{q}\left(t_{1}\right)=\tilde{\varphi_{j}^{q}}\left(t_{1}\right)-\tilde{\varphi_{i}^{q}}\left(t_{1}\right)= \\
& \frac{f}{c} \Delta p_{i j}^{q}\left(t_{1}\right)-f V_{t_{i j}}\left(t_{1}\right)-\Delta N_{i j}^{q}- \\
& \frac{f}{c} \Delta\left(V_{\text {ion }}^{t_{1}}\right)^{q}-\frac{f}{c}\left(V_{\text {tuop }}^{t_{1}}\right)_{i j}^{q}
\end{aligned}
$$


Difference of Eq.11 and Eq.12:

$$
\begin{aligned}
& \Delta \varphi_{i j}^{p q}\left(t_{1}\right)=\Delta \varphi_{i j}^{q}\left(t_{1}\right)-\Delta \varphi_{i j}^{p}\left(t_{1}\right)= \\
& \frac{f}{c}\left[\Delta p_{i j}^{q}\left(t_{1}\right)-\Delta p_{i j}^{p}\left(t_{1}\right)\right]-\left[\Delta N_{i j}^{q}-\Delta N_{i j}^{p}\right] \\
& -\frac{f}{c}\left[\Delta\left(V_{\text {ion }}^{t_{1}}\right)_{i j}^{q}-\Delta\left(V_{\text {ion }}^{t_{1}}\right)_{i j}^{p}\right] \\
& -\frac{f}{c}\left[\left(V_{\text {tuop }}^{t_{1}}\right)_{i j}^{q}-\left(V_{\text {tuop }}^{t_{1}}\right)_{i j}^{p}\right]=\frac{f}{c} \Delta p_{i j}^{p q}\left(t_{1}\right)- \\
& \Delta N_{i j}^{p q}-\frac{f}{c} \Delta\left(V_{\text {ion }}^{t_{1}}\right)_{i j}^{p q}-\frac{f}{c}\left(V_{\text {tuop }}^{t_{1}}\right)_{i j}^{p q}
\end{aligned}
$$

Because of indoor, not to think of:

$$
\frac{\mathrm{f}}{\mathrm{c}} \Delta\left(\mathrm{V}_{\text {ion }}^{\mathrm{t}_{1}}\right)_{\mathrm{ij}}^{\mathrm{pq}} \text { and } \frac{\mathrm{f}}{\mathrm{c}}\left(\mathrm{V}_{\text {tuop }}^{\mathrm{t}_{1}}\right)_{\mathrm{ij}}^{\mathrm{pq}} \text {, so }
$$$$
\Delta \varphi_{i j}^{p q}\left(t_{1}\right)=\frac{1}{\lambda} \Delta p_{i j}^{p q}-\Delta N_{i j}^{p q}
$$

Therefore:

$$
\begin{aligned}
& \Delta \rho_{i j}^{p}\left(t_{1}\right)=\rho_{j}^{p}\left(t_{1}\right)-\rho_{i}^{p}\left(t_{1}\right) \\
& =\sqrt{\left(x^{p}-x_{j}\right)^{2}+\left(y^{p}-y_{j}\right)^{2}+\left(z^{p}-z_{j}\right)^{2}} \\
& -\sqrt{\left(x^{p}-x_{i}\right)^{2}+\left(y^{p}-y_{i}\right)^{2}+\left(z^{p}-z_{i}\right)^{2}} \\
& \Delta \rho_{i j}^{q}\left(t_{1}\right)=\rho_{j}^{q}\left(t_{1}\right)-\rho_{i}^{q}\left(t_{1}\right) \\
& =\sqrt{\left(x^{q}-x_{j}\right)^{2}+\left(y^{q}-y_{j}\right)^{2}+\left(z^{q}-z_{j}\right)^{2}} \\
& -\sqrt{\left(x^{q}-x_{i}\right)^{2}+\left(y^{q}-y_{i}\right)^{2}+\left(z^{q}-z_{i}\right)^{2}}
\end{aligned}
$$$$
\Delta \rho_{\mathrm{ij}}^{\mathrm{pq}}=(16)-(15) \text {, because of the fol- }
$$

low is known:

$$
\sqrt{\left(x^{p}-x_{i}\right)^{2}+\left(y^{p}-y_{i}\right)^{2}+\left(z^{p}-z_{i}\right)^{2}}
$$$$
\sqrt{\left(x^{q}-x_{i}\right)^{2}+\left(y^{q}-y_{i}\right)^{2}+\left(z^{q}-z_{i}\right)^{2}}
$$

$$
\Delta \rho_{\mathrm{ij}}^{\mathrm{pq}} \text { is defined c。 } \Delta \mathrm{N}_{\mathrm{ij}}^{\mathrm{pq}} \text { is ambigu- }
$$
ity of whole cycles, here neglect it.

$$
\begin{aligned}
& \frac{\lambda \Delta \varphi_{i j}^{p q}+c=}{\begin{array}{l}
\text { itisconstant }, \\
\text { defined } R
\end{array}} \\
& \sqrt{\left(x^{q}-x_{j}\right)^{2}+\left(y^{q}-y_{j}\right)^{2}+\left(z^{q}-z_{j}\right)^{2}}- \\
& \sqrt{\left(x^{p}-x_{j}\right)^{2}+\left(y^{p}-y_{j}\right)^{2}+\left(z^{p}-z_{j}\right)^{2}}
\end{aligned}
$$

Then,

$$
\begin{aligned}
& R=\underline{\underline{\underline{\left(x^{q}-x_{j}\right)^{2}+\left(y^{q}-y_{j}\right)^{2}+\left(z^{q}-z_{j}\right)^{2}}}} \\
& -\frac{\sqrt{\left(x^{p}-x_{j}\right)^{2}+\left(y^{p}-y_{j}\right)^{2}+\left(z^{p}-z_{j}\right)^{2}}}{\text { defined } R_{2}} \\
& R^{2}+2 R R_{2}+R_{2}^{2}=R_{1}^{2} \Rightarrow \\
& R^{2}=R_{1}^{2}-R_{2}^{2}-2 R R_{2} \\
& =\left(x^{q}-x_{j}\right)^{2}+\left(y^{q}-y_{j}\right)^{2}+ \\
& \left(z^{q}-z_{j}\right)^{2}-R_{2}^{2}-2 R R_{2}
\end{aligned}
$$

According to Eq.17, the receiver $\mathrm{i}(\mathrm{i}=\mathrm{p}, \mathrm{m}, \mathrm{n})$ :

$$
\begin{aligned}
& \frac{\lambda \Delta \varphi_{i j}^{p q}+c_{1}}{\text { constant, defined } R}=\rho_{j q}-\rho_{j p} \\
& \frac{\lambda \Delta \varphi_{i j}^{p m}+c_{2}}{\text { constant, defined } R^{\prime}}=\rho_{j m}-\rho_{j p} \Rightarrow \\
& \frac{\lambda \Delta \varphi_{i j}^{p n}+c_{3}}{\text { constant, defined } R^{\prime \prime}}=\rho_{j n}-\rho_{j p}
\end{aligned}
$$$$
\left(x^{q}-x_{j}\right)^{2}+\left(y^{q}-y_{j}\right)^{2}+\left(z^{q}-z_{j}\right)^{2}
$$$$
-R_{2}^{2}-2 R R_{2}=R^{2}
$$$$
\left(x^{m}-x_{j}\right)^{2}+\left(y^{m}-y_{j}\right)^{2}+\left(z^{m}-z_{j}\right)^{2}
$$$$
-R_{2}^{2}-2 R^{\prime} R_{2}=R^{\prime 2}
$$$$
\left(x^{n}-x_{j}\right)^{2}+\left(y^{n}-y_{j}\right)^{2}+\left(z^{n}-z_{j}\right)^{2}
$$$$
-R_{2}^{2}-2 R^{\prime \prime} R_{2}=R^{\prime \prime 2}
$$

where $x_{j} \quad y_{j} \quad z_{j} \quad R_{2}$ are

\section{unknown quantities.}

Taylor expansion at $\left(\mathrm{x}_{0}, \mathrm{y}_{0}, \mathrm{z}_{0}\right)$,

$\mathrm{f}(\mathrm{x})=\mathrm{f}\left(\mathrm{x}_{0}\right)-\mathrm{f}^{\prime}\left(\mathrm{x}_{0}\right)\left(\mathrm{x}-\mathrm{x}_{0}\right)-\frac{\mathrm{f}^{\prime \prime}\left(\mathrm{x}_{0}\right)}{2 !}\left(\mathrm{x}-\mathrm{x}_{0}\right)^{2}+\cdots$

$f=\sqrt{\left(x^{q}-x_{j}\right)^{2}+\left(y^{q}-y_{j}\right)^{2}+\left(z^{q}-z_{j}\right)^{2}}-$

$\sqrt{\left(x^{p}-x_{j}\right)^{2}+\left(y^{p}-y_{j}\right)^{2}+\left(z^{p}-z_{j}\right)^{2}}-R$

$G=f^{\prime}=$

$\left[\begin{array}{lll}\frac{x_{1}-x_{0}}{R_{1}}-\frac{x_{2}-x_{0}}{R_{2}} & \frac{y_{1}-y_{0}}{R_{1}}-\frac{y_{2}-y_{0}}{R_{2}} & \frac{z_{1}-z_{0}}{R_{1}}-\frac{z_{2}-z_{0}}{R_{2}} \\ \frac{x_{1}-x_{0}}{R_{1}}-\frac{x_{3}-x_{0}}{R_{3}} & \frac{y_{1}-y_{0}}{R_{1}}-\frac{y_{3}-y_{0}}{R_{3}} & \frac{z_{1}-z_{0}}{R_{1}}-\frac{z_{3}-z_{0}}{R_{3}} \\ \frac{x_{1}-x_{0}}{R_{1}}-\frac{x_{4}-x_{0}}{R_{4}} & \frac{y_{1}-y_{0}}{R_{1}}-\frac{y_{4}-y_{0}}{R_{4}} & \frac{z_{1}-z_{0}}{R_{1}}-\frac{z_{4}-z_{0}}{R_{4}}\end{array}\right]$

Then, the GDOP is given by: 


$$
G D O P=\sqrt{\left(G^{T} G\right)^{-1}}
$$

According to the previously cited theorem from Ref.[4], we can easy to know that GDOP $\geq \sqrt{2}$ for four-receiver GDOP bound.

\subsection{Discussion for positioning accura- cy}

Suppose that the positioning range is the $30 \mathrm{~m} \times 20 \mathrm{~m} \times 3.5 \mathrm{~m}$ space. Receiver stations arrangement is illustrated in Fig.2. We known, GDOP expresses easiness of geometrical effect of receivers arrangement on the error. There is the higher GDOP value, the higher probability that the geometrical arrangement influences the positioning accuracy. Based on Eq.20, we can obtained the GDOP is between 2 and 5.It is assumed that there are not multi-path and fading.

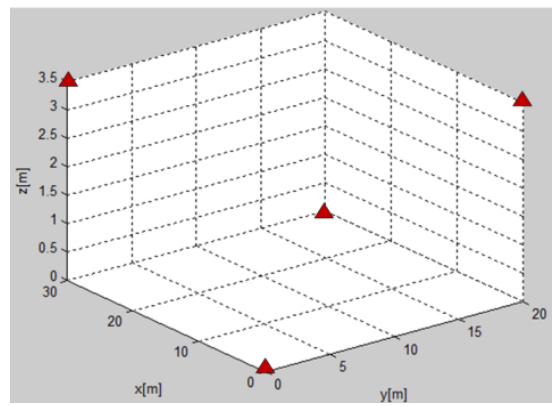

Fig.2 arrangenments of receivers

In doors, we neglect the troposphere and ionosphere error, location error of ITPS is given by:

$$
\sigma=\sqrt{\sigma_{\text {clock }}^{2}+\sigma_{\text {phase }}^{2}} \sigma_{\text {clock }} \text { is receiv- }
$$

er crystal error, we supposed it frequency is $10-10 \mathrm{~Hz}$, thus $\sigma_{\text {clock }}=0.03 \mathrm{~m} \sigma_{\text {phase }}$ is carrier phase error, if we can obtain 10 precision, then $\sigma_{\text {phase }}=5.55 \times 10^{-4} \mathrm{~m}$. $\sigma=\sqrt{0.03^{2}+\left(5.55 \times 10^{-4}\right)^{2}} \approx 0.03 \mathrm{~m}$

Then positioning error is about from $6 \mathrm{~cm}$ to $15 \mathrm{~cm}$.

\section{Conclusion}

A study on carrier phase observation principle of the indoor inverse transmitting positioning system proposed. We rigorously derived carrier phase observation equation. Because of indoors, the value of GDOP influence on positioning accuracy deeply, so in order to analyze the ITPS positioning accuracy, we also induced the formula GDOP using double difference method. Last, we discussed the positioning accuracy on condition that not to think multi-path and fading. Future works are hardware experiments using the determined scheme.

\section{References}

[1] Wang Guangyun. GPS Precise Geodetic System Principle, surveying and mapping press, Beijing, 1998.

[2] http://www.beidou.gov.cn/

[3] YU Miao, CHEN Jie, DOU Li-hua, GAN Ming-gang. Positioning accuracy of IGPS. Journal of Harbin Institute of Technology ( New series ), 17, (2), pp:219-224, 2010.

[4] Levanon N. Lowest GDOP in 2-D scenarios. IEE Proc. Radar, Sonar Navig. 2000, 147(3):149-155.

[5] Feng, C, Au, WSA, Valaee, S, Tan, $\mathrm{ZH}$, "received signal strength based indoor positioning using compressive sensing", IEEE transactions on mobile computing, DEC, 2012, 11, (12), PP. 1983-1993.

[6] Hui Liu, Houshang Darabi, Pat Banerjee, Jing Liu, "Suvey of Wireless Indoor Positioning Techniques and Systems", IEEE transactions on systems,man, and cybernetics, NOV, 2007, 37, (6), pp. 1067-1080.

[7] Ozsoy,K., Bozkurt,A., Tekin, “ indoor positioning based on global positioning system signals", microwave and optical technology letters, May, 2013, 55, (5), pp.1091-1097. 